\title{
Positron Annihilation Study of ZnO Nanoparticles Grown Under Folic Acid Template
}

Sreetama Dutta ${ }^{1}$, Sourav Sarkar ${ }^{2}$ and Bichitra Nandi Ganguly ${ }^{1 *}$

${ }^{1}$ Saha Institute of Nuclear Physics, 1/AF Bidhannagar, Kolkata, India

${ }^{2}$ IISER, Kolkata, India

\begin{abstract}
Positron lifetime spectroscopy (PAL), Doppler broadening (DB) as well as coincidence Doppler broadening (CDB) spectroscopy of a new variety of Folic acid (FA) capped zinc oxide nano-particle samples has been performed at room temperature. The results show interesting patterns of observation, hither-to unobserved in $\mathrm{ZnO}$ wurtzite crystalline samples, such as predominance of positronium formation, as reflected in the (PAL) analysis, phase transition in the nano crystalline samples at $\sim 0.8-1.0 \%$ FA concentration as depicted from DB results. Also, the chemical environment of the samples has been analysed from the ratio curves of CDB studies. Beside these, other independent results from X-ray diffraction (XRD) data and Debye- Scherrer method, Transmission electron microscopic (TEM) observations and as well as Fourier transformed infrared spectroscopic (FT-IR) analysis are reported for comparison.
\end{abstract}

Keywords: Positron annihilation spectroscopy; Folic acid templated $\mathrm{ZnO}$; Nano-particles

\section{Introduction}

Despite manifold interests on the wide band gap $(3.37 \mathrm{eV})$ semiconductor properties of zinc oxide $(\mathrm{ZnO})$ material and its varied optoelectronic applications [1,2], much remains to be explored when its material perspective is sought in the context of nanotechnology and biomedicine. $\mathrm{ZnO}$ is generally known to be nontoxic and biocompatible substance [3]. It is also realized that $\mathrm{ZnO}$ nanomaterials with the size dependent surface properties hold considerable promise as anticancer agent; the area is sensitive and important to biomedicine [4]. The field eventually leads to versatile platforms for biomedical applications and therapeutic intervention.

Growing nano sized mono dispersed $\mathrm{ZnO}$ has been one of the very challenging tasks, which we have developed in our laboratory using solgel technique, under the template of a biologically important molecule such as folic acid under physiological conditions, maintaining the $\mathrm{pH}$ of the medium and temperature [5]. The characteristic physical properties of the nano sized material so prepared under the bio-template has been examined initially with the help of various physical techniques like XRD analysis, FT-IR spectroscopy, for its purity and chemical bonding aspects [5].

But for the detail micro structural analysis and any surface vacancy related studies in the wurtzite micro crystalline $\mathrm{ZnO}$ nanomaterials, that might reveal the exact mechanism of surface related interaction or any residual charge effects which may vary under the preparation condition with respect to the particle size effects and folic acid concentration, the material has been subjected to positron annihilation spectroscopic studies (PAS), using positron life time (PAL) measurements of the samples and the Doppler broadening (DB) line shape studies with ' $S$ ' and ' $W$ ' parameter analysis. A coincidence Doppler broadening (CDB) study has been further under taken, with the help of a second (highly efficient $\mathrm{NaI}(\mathrm{Tl})$ scintillator) detector in coincidence to HPGe solid state detector to minimize the back ground effects, in order to perceive the differences with highly pure sol-gel grown $\mathrm{ZnO}$ oxide nanoparticle (wurtzite structure) sample and the bio-templated sol-gel grown sample, with respect to momentum distribution analysis (at the higher momentum side, $511 \mathrm{keV}+\Delta \mathrm{E}$ ), considering the ratio curves of the $\mathrm{CDB}$, which significantly attributes to the ensconced chemical environment [6] of the nano-particles. The results reflect interesting transition in molecular properties from the DB line shape analysis and the intriguing features in the structural properties vis- $a$ vis the analysis from PAL and CDB due to capping of the nano particles within folic acid environment. The effects are in corroboration with other complementary techniques like XRD and Debye-Scherrer method, FT-IR analysis and TEM observation which affirms the changes in structural morphology as well. Although $\mathrm{ZnO}$ nano particles have been investigated by many investigators earlier [7], but study of bio-functionalized nano particles, by PAS is sparse. The use of PAS as a micro-structural probe, for elucidation of modifications in the structural properties of $\mathrm{ZnO}$-folic acid conjugate opens a new avenue as has been delineated in this article.

\section{Experiment}

\section{Sample preparation}

$\mathrm{ZnO}$ pure nano-particles were prepared by the sol-gel technique [5] from zinc acetate (A.R. grade), in alkaline solution phase using 1:1 ammonia solution (Merck, India), at $\mathrm{pH}=7.5$. The Folic acid templated sample has been obtained using Folic acid (M.F: $\mathrm{C}_{19} \mathrm{H}_{19} \mathrm{~N}_{7} \mathrm{O}_{6}$ procured from Sigma Life Science) in the same alkaline solution at room temperature. In all cases of solution preparation, triple distilled water has been used.

\section{X-ray diffraction study}

The phase structures of the samples were identified by X-ray

*Corresponding author: Bichitra Nandi Ganguly, Saha Institute of Nuclear Physics, 1/AF Bidhannagar, Kolkata, India, Tel: 033-2337-2356; E-mail: bichitra.ganguly@saha.ac.in

Received November 18, 2013; Accepted January 16, 2014; Published January 25,2014

Citation: Dutta S, Sarkar S, Ganguly BN (2014) Positron Annihilation Study of ZnO Nanoparticles Grown Under Folic Acid Template. J Material Sci Eng 3: 134 doi:10.4172/2169-0022.1000134

Copyright: ( 2014 Dutta S, et al. This is an open-access article distributed unde the terms of the Creative Commons Attribution License, which permits unrestricted use, distribution, and reproduction in any medium, provided the original author and source are credited. 
diffraction technique using Rigaku TTRAX3 diffractometer with $\mathrm{CuKa}$ radiation (wavelength of the radiation, $\mathrm{k}=1.54 \AA$ ). The data have been collected in the range $(2 \theta) 20^{\circ}-80^{\circ}$ with a step size of $0.02^{\circ}$. Si has been used as external standard to deconvolute the contribution of instrumental broadening. The grain sizes of the synthesized samples have been calculated using Scherrer formula [8]:

$$
\mathrm{D}_{\mathrm{hkl}}=\mathrm{K} \lambda / \beta_{\mathrm{P}} \cos \theta
$$

where, $D_{\text {hkl }}$ is the average grain size, $K$ the shape factor (taken as $0.9), \lambda$ is the $X$-ray wavelength, $\beta_{\mathrm{p}}$ is the full width at half maximum (FWHM) intensity and $\theta$ is the Bragg angle. The results are shown in Figure 1 and Table 1.

\section{Transmission Electron Microscopic (TEM) study}

The morphology of the synthesized product were characterized by transmission electron microscopy, TEM (Tecnai S-twin, FEI) using an accelerating voltage of $200 \mathrm{kV}$, having a resolution of $\sim 1 \AA$. The nano crystalline grains were dispersed in isopropyl alcohol and sonicated to remove any agglomerated particles and dried under high vacuum prior to examination under TEM. The results are shown in Figure 2 and schematically explained in Figure 3.

\section{Positron annihilation spectroscopy}

Lifetime measurements: A fast-fast gamma-gamma coincidence circuit was used for the purpose of measuring positron annihilation lifetime spectrum using spectroscopic quality $\mathrm{BaF}_{2}$ as the scintillators (cone-shaped) coupled to XP2020Q photomultiplier tubes as the detectors, with the positron source $\left(\mathrm{Na}^{22}\right.$ in the form of $\mathrm{NaCl}, \sim 5 \mu \mathrm{Ci}$ point source, on an ultra thin $\mathrm{Ni}$ foil $\sim 3 \mu \mathrm{m}$ and folded) and sandwiched

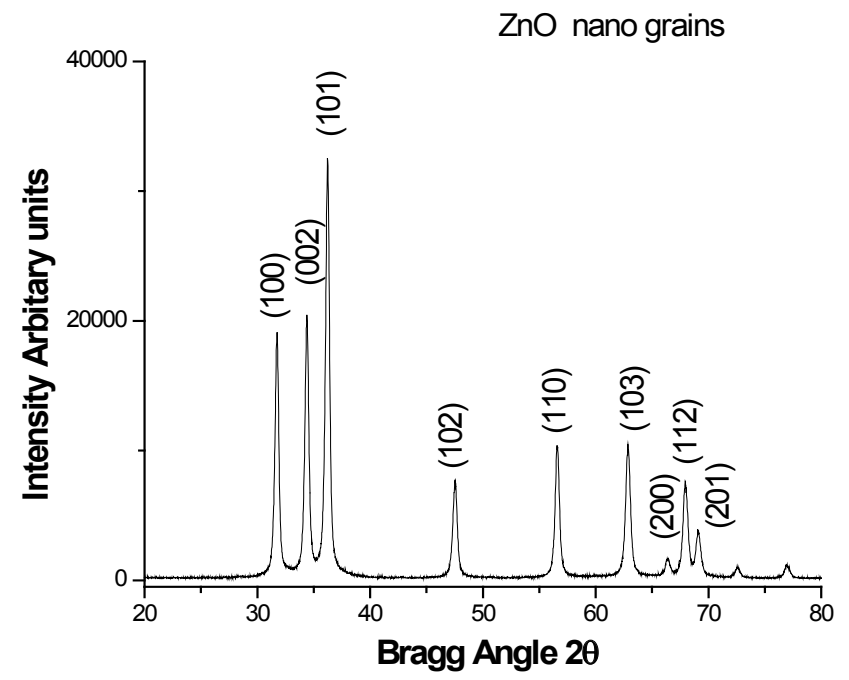

Figure 1: Chracteristic $X$-ray diffraction pattern of the synthesized nano grains of pure $\mathrm{ZnO}$ as a representative example for calculating the grain size.

\begin{tabular}{|c|c|}
\hline Samples & Size (nm) \\
\hline Pure ZnO & $\leq 10$ \\
\hline $0.5 \%$ FA & $18 \pm 1$ \\
\hline $1.3 \%$ FA & $18 \pm 1$ \\
\hline $2.0 \%$ FA & $20 \pm 2$ \\
\hline $4.8 \%$ FA & $20 \pm 2$ \\
\hline
\end{tabular}

Table 1: The Size variation of pure and FA templated $\mathrm{ZnO}$ samples as measured by Debye-Scherrer method from XRD data.

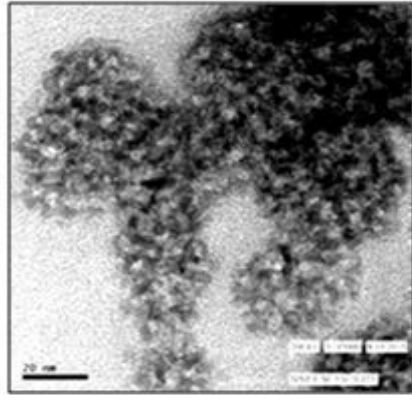

(a)

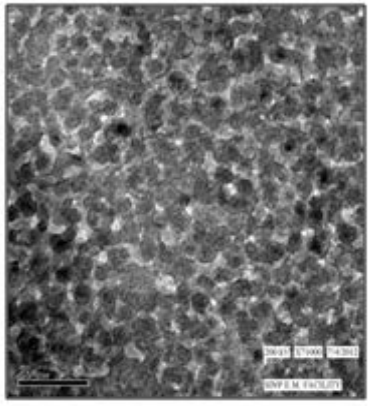

(b)

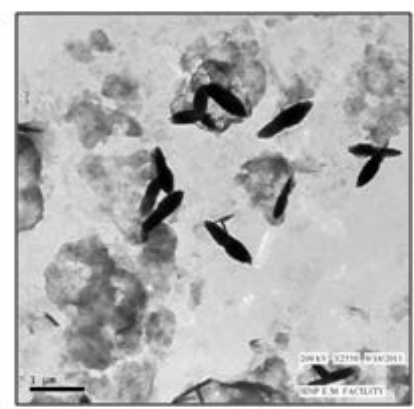

(c)

Figure 2: TEM picture representing a) Pure $\mathrm{ZnO}$ nano grains, b) folic acid ensconced $\mathrm{ZnO}-\mathrm{FA}(2 \%)$ showing swollen structure and $\mathrm{c}$ ) in presence of high concentration of folic acid, $\mathrm{ZnO}-\mathrm{FA}(4.8 \%)$ showing spindle shaped structure.

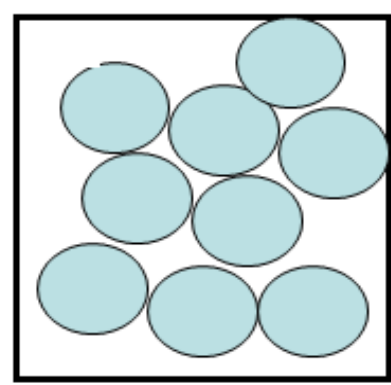

(a)

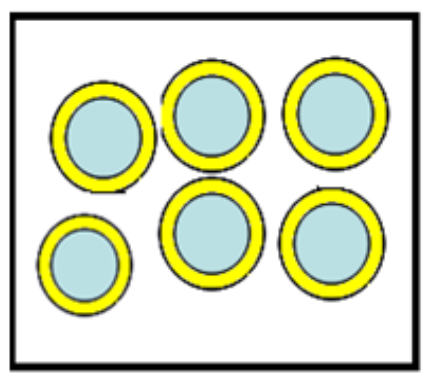

(b)

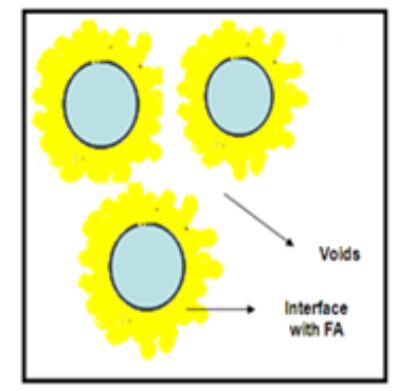

(c)

Figure 3: Schematic diagram of the a) pure $\mathrm{ZnO}$ nano grains, b) $\mathrm{ZnO}$ nano grains in coronated by the presence of the organic functional groups such as folic acid (FA), c) loosely bound structure of the $\mathrm{ZnO}$ nano particles and FA with free space. 
between the compact samples (in pallet form). A total of $10^{6}$ counts were recorded under the spectrum with a peak to back ground ratio 5000:1. The resolution of the spectrometer was $\sim 260 \mathrm{ps}$, as measured by $\mathrm{Co}^{60}$ source at the positron window settings. The spectrum was analyzed by latest version of PATFIT package program [9] namely PALSfit, with a necessary source correction which showed three life time components, the results are shown in Figures 4 and 5.

Doppler broadening (line shape) spectroscopy: The detector used in DB spectrometer is a close ended co-axial high purity $\mathrm{Ge}$ (HPGe) detector (p-type) from ORTEC. Standard sources namely $\mathrm{Co}^{60}$ with $\gamma$ photopeak at $1.33 \mathrm{MeV}$, the energy resolution (FWHM $\sim 1.75 \mathrm{keV}$ ), and with $\mathrm{Bi}^{207}$ for the $\gamma$ photopeak at $579 \mathrm{keV}$ with the energy resolution (FWHM $\sim 1.1 \mathrm{keV}$ ) were used for calibration. The experimental spectrum was recorded around $511 \mathrm{keV}$ annihilation line in order to determine the 'S' parameter, this being evaluated as the ratio of counts in the central region $(511 \pm 1.7 \mathrm{keV})$ of the spectrum to the total counts recorded under the spectrum. The results are represented in Figure 6. Also 'W' parameter was measured at the wings $(2 \mathrm{keV}<|\mathrm{E}-511|<7 \mathrm{keV})$ for the different samples on either side of the Doppler spectrum to the total fixed counts under the spectrum. The ratio of the S-W plot is presented in Figure 7.

Coincidence Doppler broadening spectroscopy: This technique essentially uses another detector kept at $180^{\circ}$ with the direction of the gamma rays by the original $\mathrm{HPGe}$ detector, so that the other gamma ray is detected in the second detector (in this case 3 " $\times 3$ " $\mathrm{NaI}(\mathrm{Tl})$ scintillator is used for its good efficiency) in coincidence and is used to gate a gated biased amplifier through which the amplifier output signals are fed to the multi channel analyser (Figure 8). This arrangement essentially reduces the background at the higher energy side and thus improves the peak to background ratio (5000:1), also reduces the

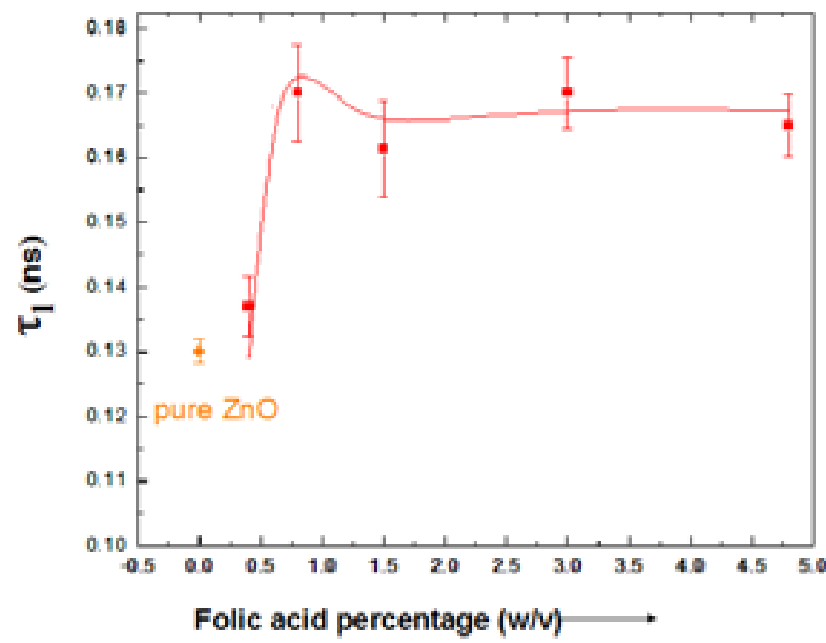

a)

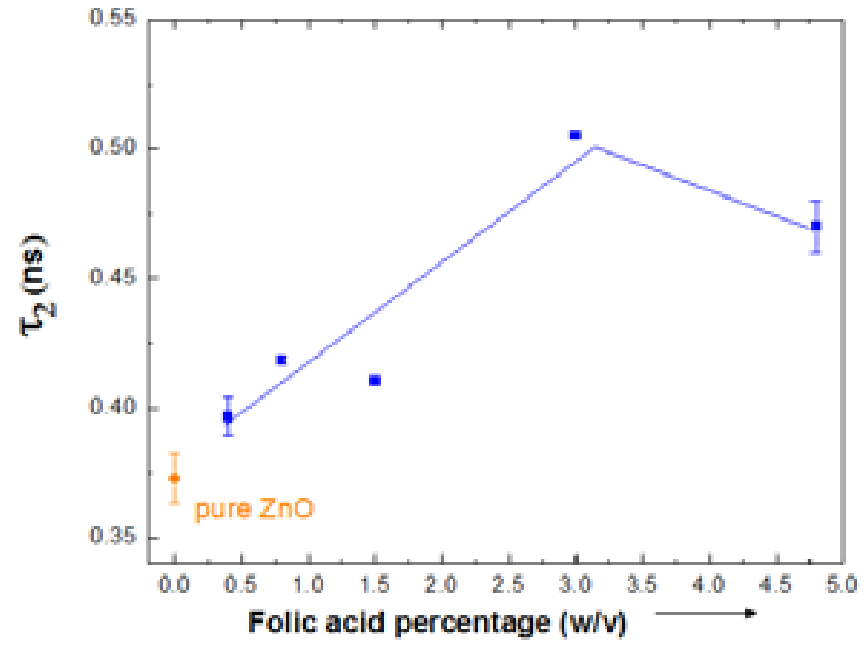

b)

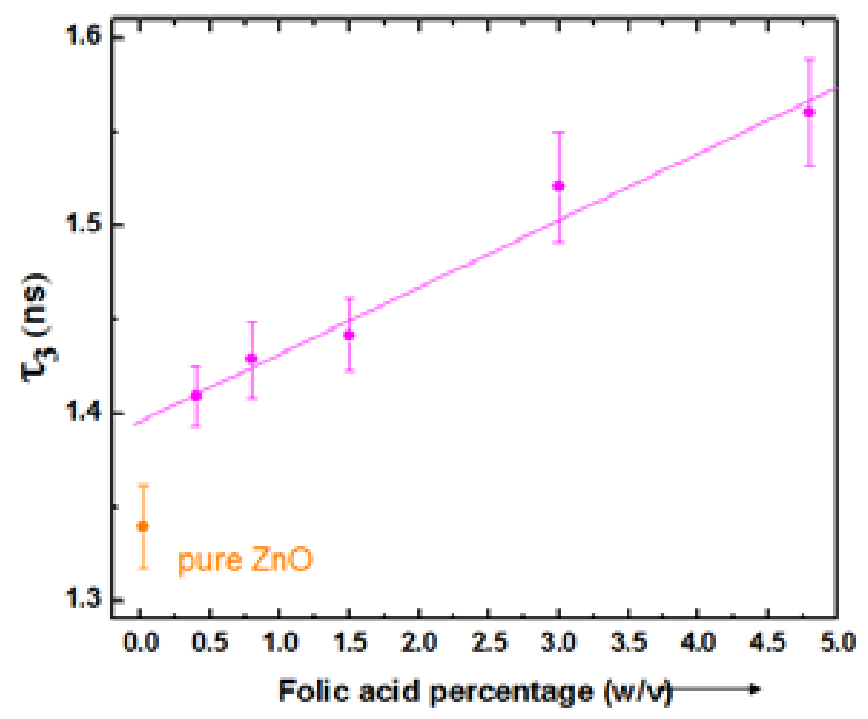

C)

Figure 4: Variation of positron life time results in $\mathrm{ZnO}$ nano grains in the presence of the variation of folic acid (FA \%) ornamented structure, a) $\mathrm{T}_{1}$, b) $\mathrm{T}_{2}$, C) $\mathrm{T}_{3}$. 


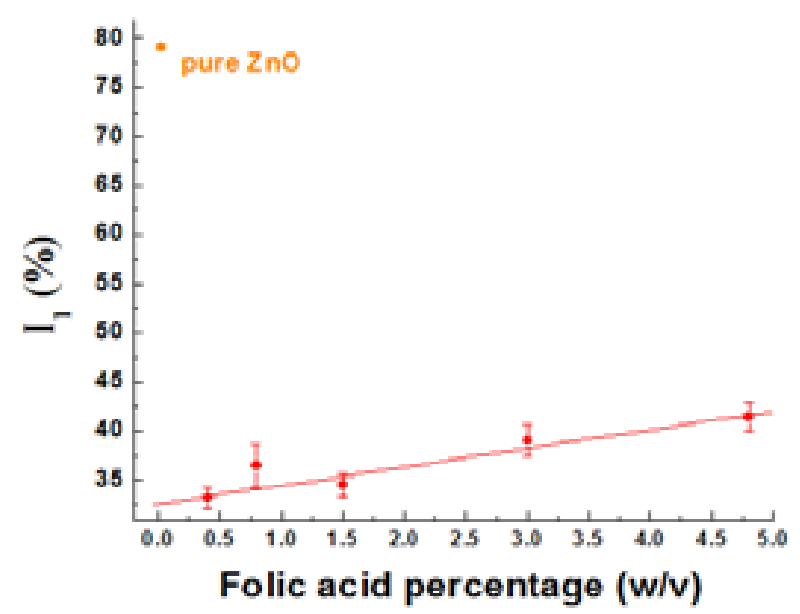

a)

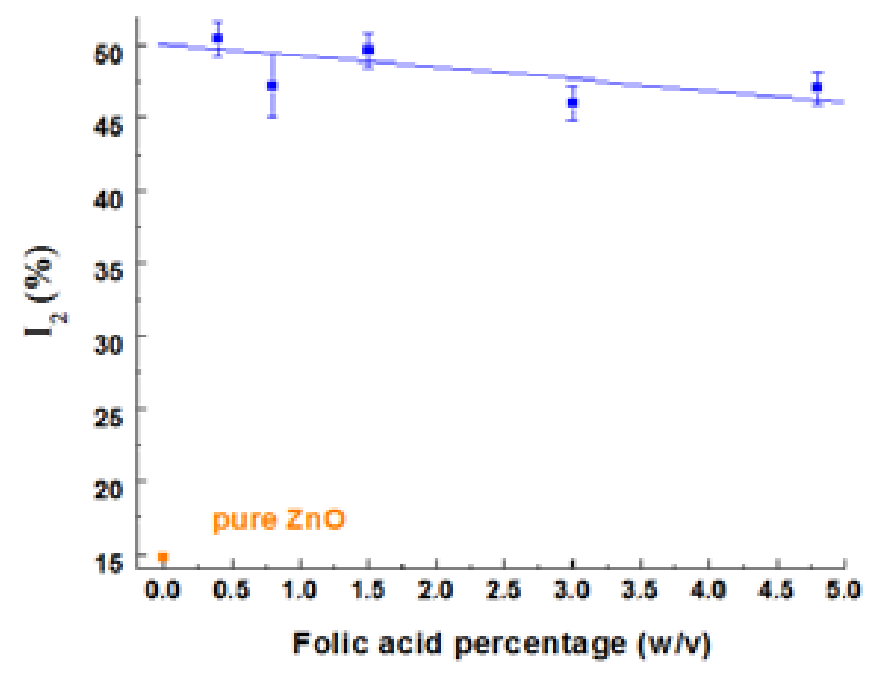

b)

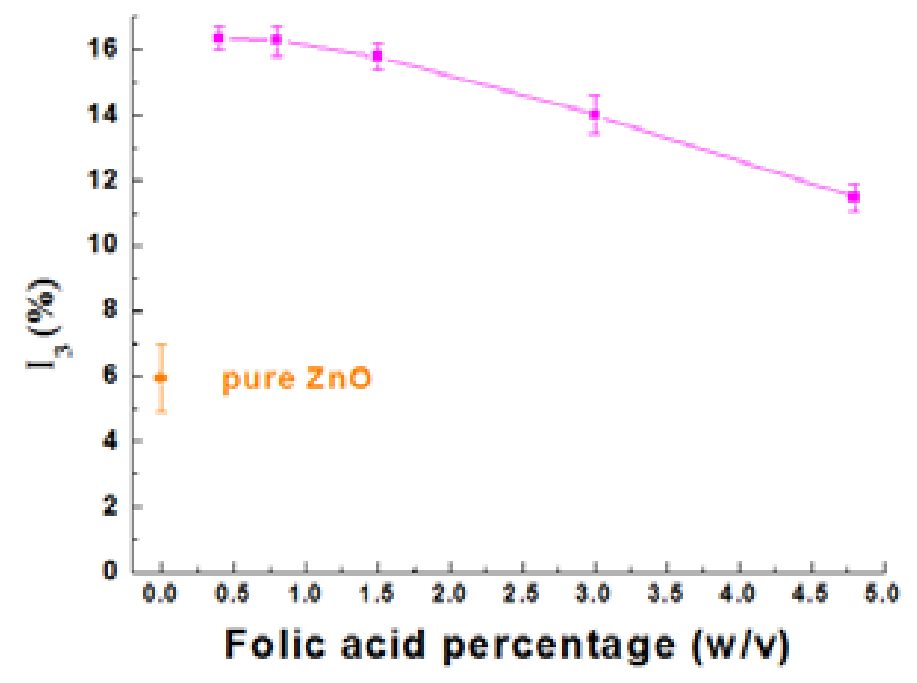

c)

Figure 5: Variation of relative percentage intensities of different positron life time components: a) $I_{1}$, b) $I_{2}$, c) $I_{3}$ in the presence of variation of FA \% in the ZnO nano crystallites preparation stages.

energy resolution by a factor $\sim \sqrt{ } 2$ such that at the wings especially at the right hand side of the spectrum $(511 \mathrm{keV}+\Delta \mathrm{E})$, the higher momentum distribution is perceived very clearly [10] after back ground subtraction and peak normalization with the reference standard sample taken as pure $\mathrm{ZnO}$. The ratio curves of $\mathrm{ZnO}-\mathrm{FA}$ / pure $\mathrm{ZnO}$ were generated, as shown in Figure 9.

\section{FT-IR Spectroscopic measurements}

Fourier Transmission Infrared (FT-IR) spectra of the powders (as pellets in $\mathrm{KBr}$, without moisture) were recorded using a Fourier Transform Infrared Spectrometer (Perkin Elmer FTIR system; Spectrum GX) in the range of $400-6000 \mathrm{~cm}^{-1}$ with a resolution of 0.2 $\mathrm{cm}^{-1}$. The results are shown in Figure 10.

\section{Result and Discussion}

\section{Size and morphology}

Sol-gel grown $\mathrm{ZnO}$ oxide nano particles offer high grade purity of the samples as well as nearly monodispersed particles (controlled size distribution) with only proper treatment with the capping agent, the excess of the same could be washed -off at the end. The result of such a synthesized sample is presented through Figure 1 and Table 1 and through the TEM micrographs in Figure 2. Although the physical characteristics and the wurtzite structure of the pure $\mathrm{ZnO}$ nano crystalline samples and the FA capped $\mathrm{ZnO}$ nano particles have been documented [5], however the size of these nano particles vary according to the concentration of the FA used in the medium, 


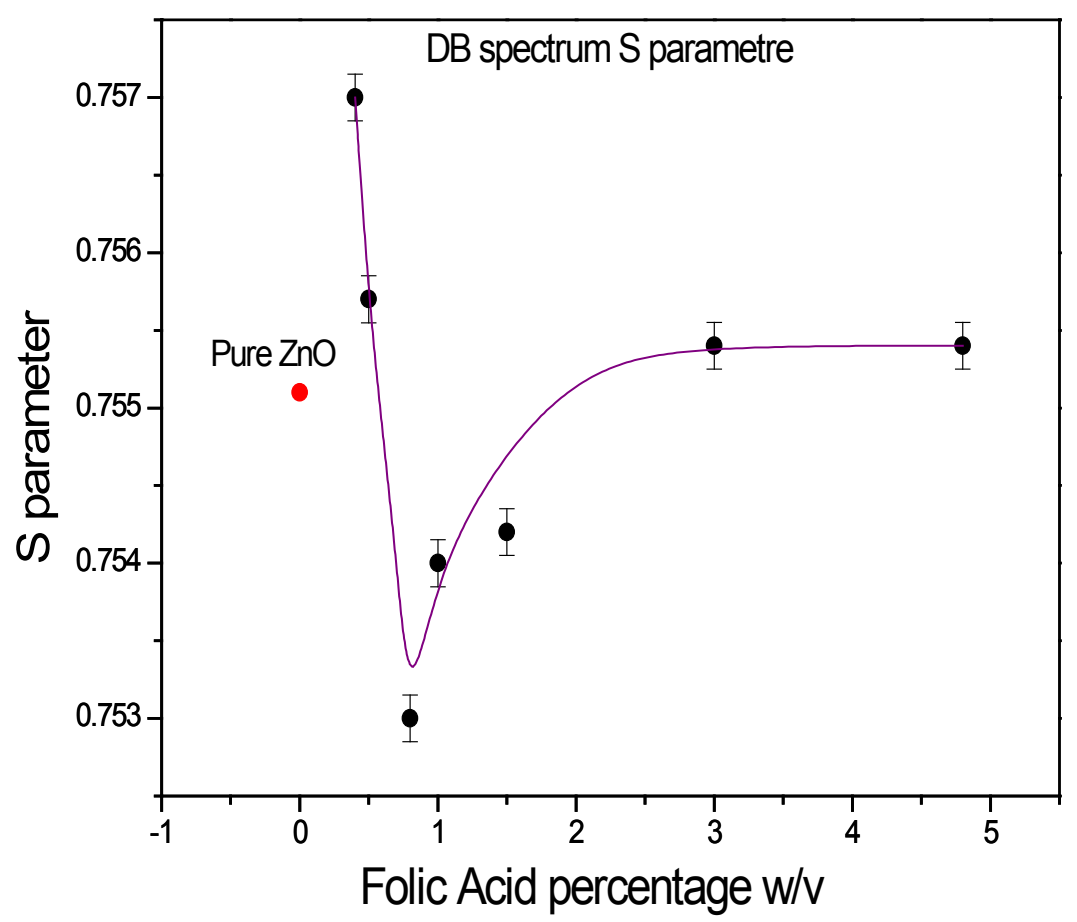

Figure 6: Doppler broadened line shape analysis: Variation of ' $S$ ' parameter showing a transition point, in the variation of electronic structure in the presence of variation of $\mathrm{FA} \%$.

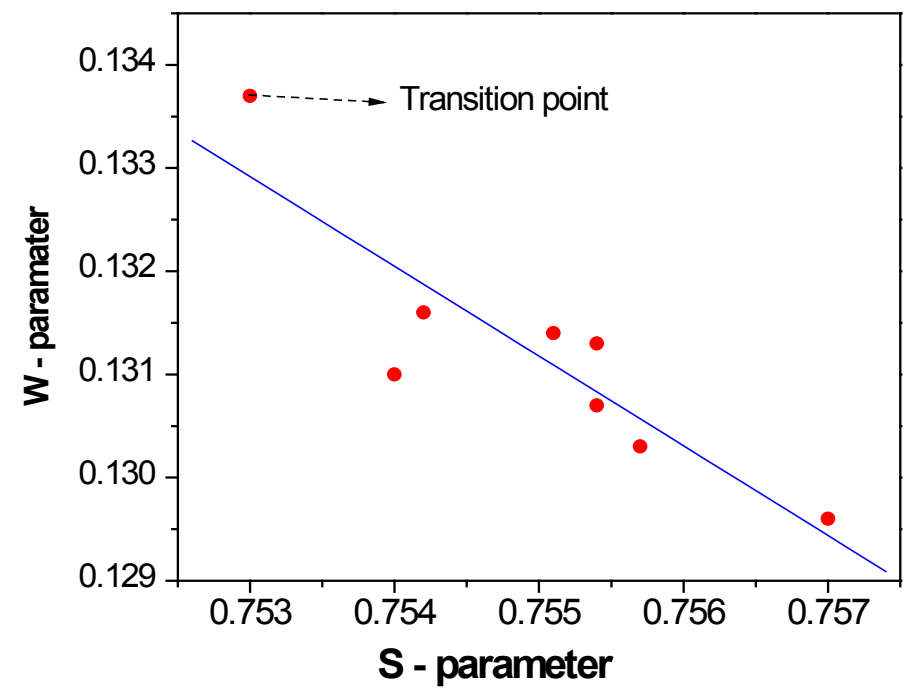

Figure 7: Ratio of S-W parameter in ZnO-FA structure representing similar type of defects, introduced in the system, the grouping points near transition are encircled, but the exact transition point stands alone at the top.

the time taken for growing and isolation of the particles while under preparation. A partial crystallinity [5] was observed with increase in concentration of FA to $4.8 \%$ [5]. The purpose of addition of FA in extremely small amounts was to control the size of the nano particles and to achieve monodispersity of the synthesized particles. It has been found that freshly prepared solution and immediate isolation of the as grown particles offer better results. The sonication of the particles and drying immediately on the microscopic carbon coated grids under high vacuum showed the particles to be less than $10 \mathrm{~nm}$. In the electron micrograph, in average particle size of $\leq 10 \mathrm{~nm}$ can be observed for pure $\mathrm{ZnO}$. But the FA ensconced $\mathrm{ZnO}$ nano particles show although a swollen structure $\sim 11 \mathrm{~nm}$ and the particles are properly isolated. An illustration of the bare nano particles and FA templated nano particles can be found in Figure 3. The excess addition of FA show an encapsulated spindle shaped structure which are much greater in size. There is however a variation in size, as shown in Table 1 as compared to Figure 2. This could be due to sonication and the drying processes involved and the inherent variations of the detection methods. 
Figure 8: Coincidence Doppler broadening (CDB) circuit diagram with two detectors, i) $40 \mathrm{cc}$ HPGE solid state , ii) Nal (TI) $3^{\prime \prime} \times 3$ " scintillator detector kept at $180^{\circ}$ opposite direction to receive the Two gamma rays at opposite direction.

\section{Micro structural analysis}

In order to investigate the detail micro structural analysis and any surface vacancy related studies in the wurtzite nano-crystalline $\mathrm{ZnO}$ grains, that might reveal the exact mechanism of surface interaction or any residual charge effects which may vary under the preparation condition, with respect to the particle size effects and folic acid concentration, the material has been subjected to positron annihilation studies using PAS, DB as well as CDB method. The qualitative variation in the positron life time parameters in the samples denotes the variation of defect related properties or any structural variation in the material as positron parameters are sensitive to any kind of electronic microenvironment. In the case of FA templated $\mathrm{ZnO}$ nano particles, results of the positron life time analysis could be fitted to three components, along with their respective intensities from the three different electronic states, including the case of pure $\mathrm{ZnO}$ sample (standard), which is has been shown in Figure 4 and 5.

The shortest life time is represented by $\tau_{1}$. The bulk life time [11] of positrons, $\tau_{b}$, of the pure $\mathrm{ZnO}$ sample has been calculated to be $\sim 153 \mathrm{ps}$ and is in accordance with the literature [12] value. However, as the FA percentage increases in the system, the $\tau_{1}$ show a change in the trend, since at this stage of preparation, due to capping, the surface state and trapping rates become important. With $\sim 1 \% \mathrm{FA}$, a transition has been noticed. Initially since $\tau_{1}<\tau_{b}(\sim 0.153 \mathrm{~ns})$, a fraction of positrons could be trapped with negatively charged $\mathrm{Zn}^{+2}$ monovacancy defects [13]. The trapped positrons' life time is expected to be larger than $\tau_{b}$, depending up on the electron density at that defect site. The trapping rate is however found to be very weak, and of the order as: $k=1.8$ (ns) $)^{-1}$; with effective annihilation rate as $\lambda_{e f f}=8.33(\mathrm{~ns})^{-1}$. Assuming a diffusion coefficient $\sim 10^{-5} \mathrm{~cm}^{2} \mathrm{~S}^{-1}$ for positrons [14], there by the diffusion length was found to be $\leq 10 \mathrm{~nm}$, which could be just at grain boundary or surface region, or even diffuse out towards low electron density region coronated by FA as shown in Figure 3.when the actual $\mathrm{ZnO}$ grain sizes are smaller.

The corresponding $\mathrm{I}_{1}$ percentages (Figure $5 \mathrm{a}$ ) from this state have been found to increase, with FA in the system, as more and more of the positrons could be trapped at this site, either for neutralizing the defects or it is possible that interaction of FA as electron donor at the nano grain surface enhances such a possibility, where positrons could be drawn. Thus, effectively we find surface charge effect has been noticeable.

The second life time component $\tau_{2,}$ varies between 370-470ps (Figure $4 \mathrm{~b}$ ), could be assigned to higher order defects at the surface or interfacial region (Figure $3 \mathrm{~b}$ ), depending up on the structural condition as the morphology varies within the system. In the nano sized particles the surface/volume ratio increases and in general the molecules are loosely bound, therefore annihilation at the surface/interface region could give rise to increase in the $\tau_{2}$ values. The concentration of FA at the interface at higher concentration (at 4.8\% FA) shows a drastic decreasing trend due to close packing, this is also discernible from the micrograph (Figure 2c). 


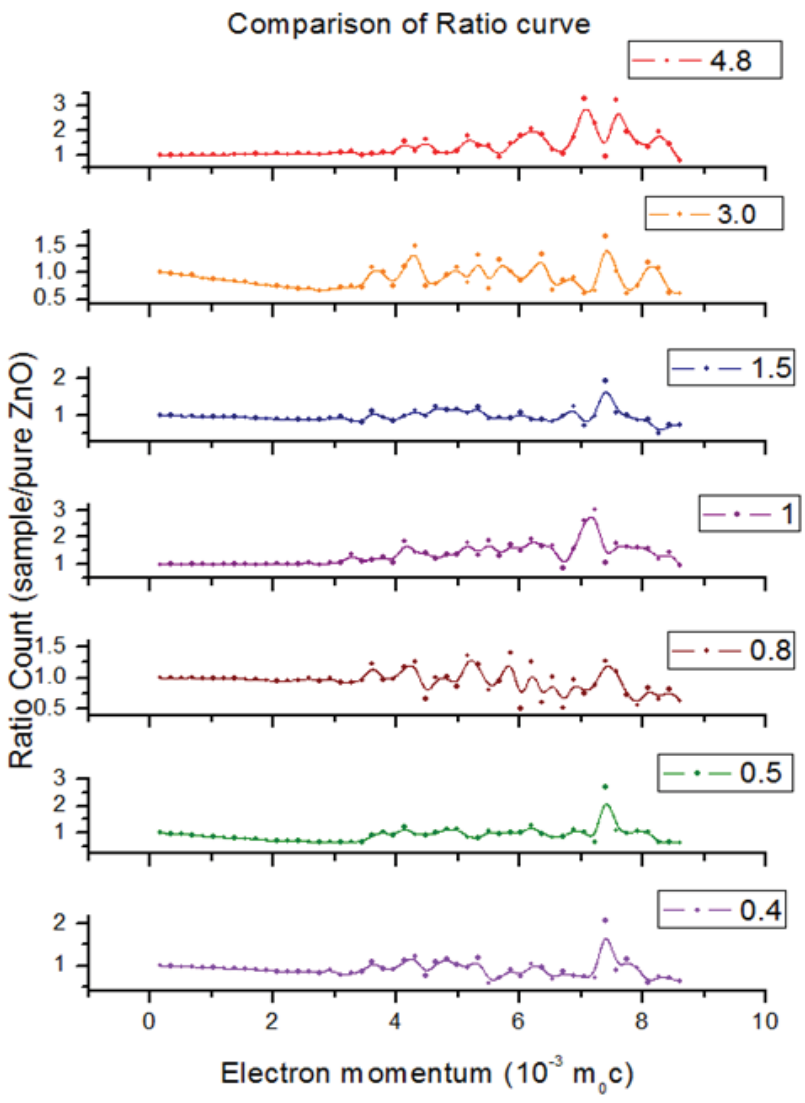

(a)

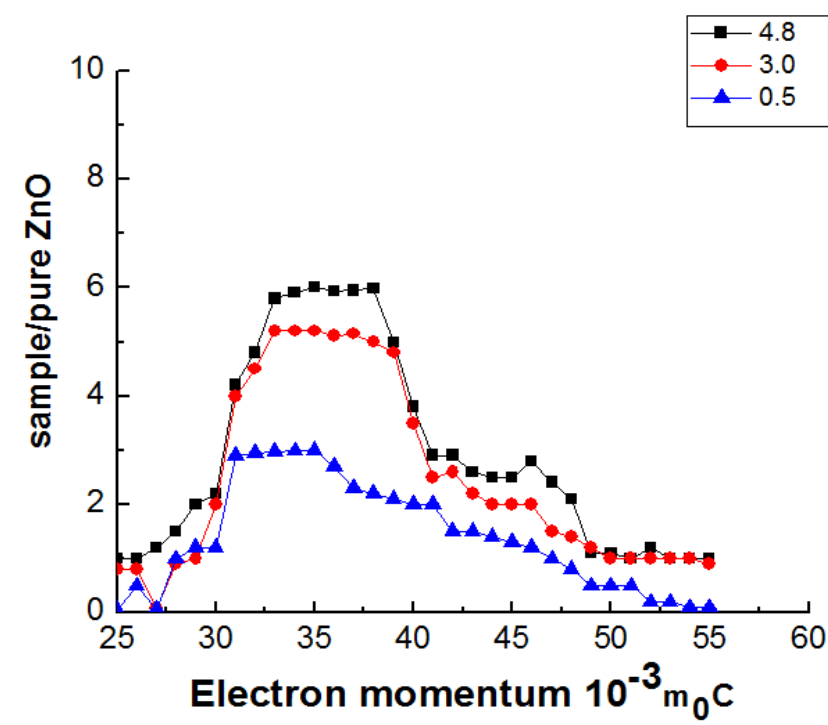

(b)

Figure 9: Analysed results of $\mathrm{CDB}$, ratio of counts in the region $(511 \mathrm{keV}+\Delta \mathrm{E})$ vs angular momentum distribution, with different $\mathrm{FA} \%$ capped $\mathrm{ZnO}$ system: a) for valance band contributed region, that is detected with $\mathrm{ZnO}-\mathrm{FA}, \mathrm{b}$ ) in the higher momentum region signifying the core electrons due to functional groups of $\mathrm{FA}$, in $\mathrm{ZnO}-\mathrm{FA}$ grains, using $\mathrm{ZnO}-$ pure as the standard spectrum.

$I_{2}$ percentage shows more or less uniform pattern within (Figure $5 b)$ the FA conjugated structure, A levelling-off of the same is due to the strongly ensconced nature of the nano particles as the FA concentration increases in the system (Figure $4 \mathrm{~b}$ ). This also corroborates with the morphological changes as observed by TEM (Figure 2).

A profound effect is that of positronium (Ps) formation in the system that is reflected by the third long life time component (in ns) (Figure $4 \mathrm{c}$ and $5 \mathrm{c}$ ), which is a dominant feature of the system, it is greater than $5 \%$ , wherever FA has been used, since free volume structure increases with addition FA in the system. These results also commensurate with the change of the morphology of the system, studied by Figure 2 and the illustration of the same in Figure 3. Intensity shows a decreasing trend at higher concentration of FA. This could be simply due to density effect of FA, i.e. increase in the number of molecules of FA that results in the strongly ensconced structure of the nano particles (spindle shaped structure in Figure 2) as percentage of the same increases at the preparative stages.

The momentum distribution of the annihilation radiation has been shown by the DB results in Figure 6. A structural transition in the nano particle system has been observed at $0.8-1 \%$ FA capped system from DB results through $S$ - parameter. As the surface structure gets modified (S-parameter is sensitive to valance electron states), substrate is comparatively less densely packed as FA concentration gradually reaches a critical value, $S$ parameter increases with the open volume defect enriched state (compare with Figure 4 and 5 of the positron life time results) but further with increase in concentration of FA the effect levels-off. This observation of abrupt change in line shape parameter denotes a structural transition which is also in accordance with our earlier results [5] as also the same is reflected with the $\tau_{1}$ values in PAL. $\mathrm{W}$-parameter is also measured, the $\mathrm{S} / \mathrm{W}$ ratio shows a linear fitting curve (Figure 7), denoting a uniform trend of defect structure, but as shown by the arrow, the transition point stands alone at the top, denoting a different structural characteristic feature.

At the high momentum wings of the $\mathrm{DB}$ line shape analysis, because of the low background effect due to $\mathrm{CDB}$ system with an additional detector vide (Figure 8), there exists the characteristic signature of the chemical environment due to capping effect of FA. This effect can be best perceived by the ratio curves of CDB, when the back ground effect is minimized, Figure 9 shows the signature of

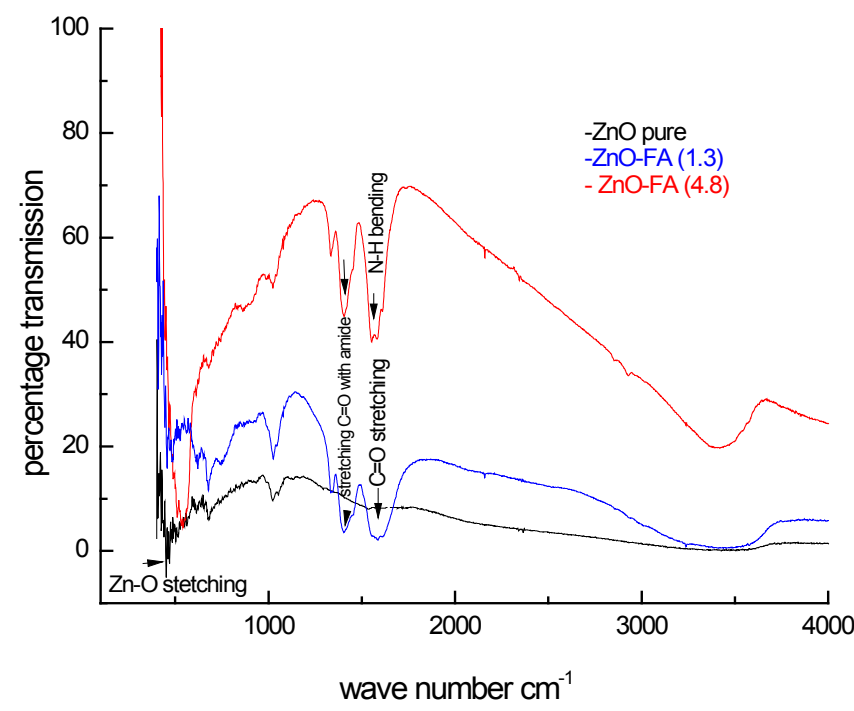

Figure 10: FT-IR characteristic absorption frequencies for the as prepared $\mathrm{Zn}$ FA nano grains with varying FA\% used in the system. 


\begin{tabular}{|c|l|}
\hline Wave number $\left.\mathbf{~ m}^{-1}\right)$ & Absorption band \\
\hline$\sim 3400-3600$ & stretching $\mathrm{O}-\mathrm{H}$ mode, $\mathrm{N}-\mathrm{H}$ mode \\
\hline$\sim 1600$ & $\begin{array}{l}\text { symmetric } \mathrm{C}=\mathrm{O} \text { stretching mode, stretching } \mathrm{CONH}_{2}, \mathrm{~N}-\mathrm{H} \\
\text { bending vibration }\end{array}$ \\
\hline$\sim 1380$ & asymmetric $\mathrm{C}=\mathrm{O}$ stretching mode \\
\hline$\sim 500$ & Zn-O stretching mode \\
\hline
\end{tabular}

Table 2: The characteristic IR frequencies $\left(\mathrm{cm}^{-1}\right)$ of $\mathrm{FA}$ as observed with the $\mathrm{ZnO}$ nano particles.

chemical environment due to FA through $2 \mathrm{~s}, 2 \mathrm{p}$ electrons of carbon and nitrogen in the co-ordinating groups like $-\mathrm{COOH}$ and $-\mathrm{NH}_{2}(\sim$ I.P. $\sim 9.4 \mathrm{eV}$ [14], binding energies $\sim 10 \mathrm{eV}$ that could correspond to a $\mathrm{DB}$ energy of $2.5-3 \mathrm{keV}$ ), the signature of which towards the right wing side of the spectrum $(511 \mathrm{keV}+\Delta \mathrm{E})$ can be significantly discernible from the improved energy resolution of the detector and due to very low back ground [10]. Really, at the high momentum side the signature of the core momentum, arising in the range of $\sim 35-40 \times 10^{-3} \mathrm{~m}_{0} \mathrm{C}$ (due to $1 \mathrm{~S}$ electrons of carbon) has been found at the far end, as the signature of core electrons from the ligands of the organic functional $(\mathrm{C}=\mathrm{O})$ group ornamenting the $\mathrm{ZnO}$ nano particles [14].

Concomitantly, presence of such chemical environment at the surface of the nano particles prepared in the template of FA could be also decided almost independently from the IR spectroscopic study of the material as shown in Figure 10 and Table 2. This characteristic emblem of FA is very important as the same makes $\mathrm{ZnO}$ nano particles bio-chemically important for targeted drug delivery purposes [4]. In this aspect, positron annihilation parameters have been shown to serve as a surface sensitive probe for the assay of the micro structural aspects of nano particles.

\section{Conclusion}

Study of $\mathrm{ZnO}$ nano particles of size less than $10 \mathrm{~nm}$ is feasible through positron annihilation spectroscopy, that senses the defects mostly at the surface and brings up the nature of surface involved in the template, along with the elemental features of the coordinating chemical group, clearly discernible from $\mathrm{CDB}$ studies. There is an onset of a structural transition in the presence of around 1\% FA in the preparation system of the $\mathrm{ZnO}$ nano grains as is indicated in $\mathrm{DB}$ studies.

\section{Acknowledgement}

The authors acknowledge the technical help received from Soma Roy for all spectroscopic data recording and Pulak Roy for TEM pictures.

\section{References}

1. Tang ZK, Wong GKL, Yu P, Kawasaki M, Ohtomo A, et al. (1998) Roomtemperature ultraviolet laser emission from self-assembled $\mathrm{ZnO}$ microcrystallite thin films. Appl Phys Lett 72: 3270-3272.

2. Zhao Z, Lei W, Zhang X, Wang B, Jiang H (2010) ZnO-Based Amperometric Enzyme Biosensors. Sensors 10: 1216-1231.

3. Salata OV (2004) Applications of nanoparticles in biology and medicine. J Nanobiotechnology 2: 3.

4. Rasmussen JW, Martinez E, Louka P, Wingett DG (2010) Zinc oxide nanoparticles for selective destruction of tumor cells and potential for drug delivery applications. Expert Opin Drug Deliv 7: 1063-1077.

5. Dutta S, Ganguly BN (2012) Characterization of ZnO nanoparticles grown in presence of folic acid template. J Nanobiotechnology 10: 29.

6. MacDoland JR, Lynn KG, Boie RA, Robbins MF (1978) A two-dimensional Doppler broadened technique in positron annihilation. Nucl Instrum Methods 153: $189-194$

7. Ghoshal T, Biswas S, Kar S, Chaudhuri S, Nambissan PM (2008) Positron annihilation spectroscopic studies of solvothermally synthesized $\mathrm{ZnO}$ nanobipyramids and nanoparticles. J Chem Phys 128: 074702.

8. Cullity BD, Stock SR (2001) Elements of X-ray Diffraction. ( $3^{\text {rd }}$ edn), PrenticeHall, New Jersey, USA.

9. http://palsfit.dk/risoe-m-2740.pdf

10. Asoka-Kumar P, Alatalo M, Ghosh VJ, Kruseman AC, Nielsen B, et al. (1996) Increased Elemental Specificity of Positron Annihilation Spectra. Phys Rev Lett 77: 2097-2100.

11. Dupasquier A, Mills Jr. AP, (1995) Positron Spectroscopy of Solids Proceedings of the International School of Physics "Enrico Fermi". IOS Perss The Netherlands.

12. Brauer G, Anwand W, Skorupa W, Kuriplach J, Melikhova O, et al. (2007) Comparative characterization of differently grown $\mathrm{ZnO}$ single crystals by positron annihilation and Hall effect. Superlattices Microstruct 42: 259-264.

13. Saarinen K, Suski T, Grzegory I, Look DC (2001) Thermal stability of isolated and complexed Ga vacancies in GaN bulk crystals. Phys Rev B 64: 233201.

14. Brandt W, Paulin R (1972) Positron Diffusion in Solids. Phys Rev B 5: 24302435. 